\title{
Recurrence Relations and Asymptotics of Colored Jones Polynomials
}

\author{
A. I. Aptekarev ${ }^{1 *}$, T. V. Dudnikova ${ }^{1 * *}$, and D. N. Tulyakov ${ }^{1 * * *}$ \\ (Submitted by A. M. Elizarov) \\ ${ }^{1}$ Keldysh Institute of Applied Mathematics, Russian Academy of Sciences, Moscow, 125047 Russia \\ Received June 17, 2021; revised June 25, 2021; accepted July 5, 2021
}

\begin{abstract}
We consider $q$-difference equations for colored Jones polynomials. These sequences of polynomials are invariants for the knots and their asymptotics plays an important role in the famous volume conjecture for the complement of the knot to the $3 \mathrm{~d}$ sphere. We give an introduction to the theory of hyperbolic volume of the knots complements and study the asymptotics of the solutions of $q$-recurrence relations of high order.
\end{abstract}

DOI: $10.1134 / \mathrm{S} 1995080221110056$

Keywords and phrases: knots, colored Jones polynomial, asymptotics of solutions to a $q$-recurrence equation.

\section{INTRODUCTION}

At first we give some definitions concerning knots from the paper [52] and the books [1, 9, 49].

A knot in 3 -sphere $\mathbb{S}^{3}$ is a continuous embedding of a topological circle in 3-dimensional space considered up to continuous deformation (isotopy). The simplest knot of all is unknotted circle, which we call the unknot or the trivial knot. The next simplest non-trivial knot is called a trefoil knot. A link is a collection of knots which do not intersect, but which may be linked together. An oriented link $L$ is a compact 1-dimensional oriented smooth submanifold of $\mathbb{R}^{3} \subset \mathbb{S}^{3}$. A framed oriented link $L$ is a link equipped with a smooth normal vector field $V$, which is a function $V: L \rightarrow \mathbb{R}^{3}$, such that $V(x)$ is not in the tangent space $T_{x} L$ for every $x \in L$.

A hyperbolic knot $K$ in $\mathbb{S}^{3}$ is defined to be a knot such that the complement $\mathbb{S}^{3} \backslash K$ is hyperbolic 3-manifold. Note that $\mathbb{S}^{3} \backslash K$ is a finite volume but noncompact hyperbolic 3-manifold (see [1]). A torus knot is a knot which can be placed on an ordinary torus in $\mathbb{S}^{3}$. With any nontrivial knot $K$ there is associated a whole collection of other knots, known as satellites of $K$; these are knots which are obtained by a nontrivial embedding of a circle in a small solid torus neighborhood of $K$. Here, "nontrivial" means that the embedding is not isotopic to $K$ itself and is not contained within a ball inside the solid torus. A knot is a satellite knot if it is a satellite of a nontrivial knot, see also [40, Definition 1.2].

The famous Thurston's theorem [52, Section 2.5] states that every knot in $\mathbb{S}^{3}$ is either a torus knot or a satellite knot or a hyperbolic knot, see also [51].

In algebraic topology, a way of studying knots is to associate some invariants, which are useful to separate knots. In the given paper, we consider one of such invariants. In 1985, Jones introduced his celebrated polynomial invariant, the Jones polynomial $J_{K}(q)$ associated to a knot $K$ [26, 27]. At first, this polynomial was defined by using the operator algebra, namely, the Yang-Baxter operator and the $R$-matrix. Shortly after, another version of this invariant was given by using the Kauffman bracket [31]. Colored versions of these invariants were defined, via quantum groups [47]. For details on the definitions of the colored Jones polynomial, see, e.g., [40, Chapter 2].

\footnotetext{
${ }^{*}$ E-mail: aptekaa@keldysh.ru

${ }^{* *}$ E-mail: tdudnikov@mail.ru

*** E-mail: dntulyakov@gmail.com
} 
Definition 1 [13]. (i) The colored Jones function $J_{K}^{\prime}: \mathbb{N} \rightarrow \mathbb{Z}\left[q^{ \pm 1}\right]$ of a knot $K$ in 3-space is a sequence of Laurent polynomials whose $n$th term $J_{K}^{\prime}(n)$ is the Jones polynomial of a knot $K$ with the $n$ dimensional irreducible representation $\mathfrak{s l}_{2}$. We normalize the Jones function so that the value for the trivial knot (unknot) is one, $J_{\text {unknot }}^{\prime}(n)=1$ for all $n$.

(ii) We denote by $J_{K}: \mathbb{N} \rightarrow \mathbb{Z}\left[q^{ \pm 1 / 2}\right]$ the colored Jones function with normalization by

$$
J_{\text {unknot }}(n)=[n]:=\frac{q^{n / 2}-q^{-n / 2}}{q^{1 / 2}-q^{-1 / 2}} .
$$

Hence, $J_{K}(n)=[n] J_{K}^{\prime}(n)$. Below we use also notation: $J_{K} \equiv J_{K}(n) \equiv J_{K}(n, q)$. For any knot, $J_{K}(1)=1$ and $J_{K}(2)$ coincides with the Jones polynomial of a knot $K$.

For a detailed discussion on the polynomial invariants of knots that come from quantum groups, see, e.g., $[58,59]$.

A new approach to the study of the colored Jones function was proposed by Garoufalidis and Lê [12] by using a $q$-difference equation.

Definition 2. A $q$-difference equation for a sequence $\{f(1), f(2), \ldots\}$ of smooth functions of $q$ has the form

$$
\sum_{j=0}^{d} b_{j}\left(q^{n}, q\right) f(n+j, q)=0,
$$

where $b_{j}(u, v)$ are smooth functions and $f(n, q)=f(n)(q)$. The characteristic polynomial (see [14, Definition 1.1]) of (2) is

$$
P(v, \lambda)=\sum_{j=1}^{d} b_{j}(v, 1) \lambda^{j} .
$$

Theorem 1 [12, Theorem 1]. The colored Jones function of any knot satisfies a q-difference equation.

Recall that a sequence $f: \mathbb{N} \rightarrow \mathbb{Q}[q]$ is $q$-holonomic if it satisfies a $q$-linear difference equation, i.e., there exist a number $d \in \mathbb{N}$ and polynomials $b_{j}(u, v) \in \mathbb{Q}[u, v]$ for $j=0,1, \ldots, d$ with $b_{d} \neq 0$ such that for all $n \in \mathbb{N}$ the sequence $f$ satisfies (2). Thus, Theorem 1 states that the colored Jones function is $q$-holonomic.

Holonomic functions and systems were first introduced and studied by Bernstein [7, 8]. The algorithmic significance of $q$-holonomic sequence was first recognized by Zeilberger [62]. To prove Theorem 1, Garoufalidis and Lê showed (see [12, Proposition 3.7]) that the colored Jones function can be written as a multisum

$$
J_{K}(n)=\sum_{k_{1}, \ldots, k_{r}=0}^{\infty} F\left(n, k_{1}, \ldots, k_{r}\right)
$$

of a proper q-hypergeometric function $F\left(n, k_{1}, \ldots, k_{r}\right)$ (see [12, Definition 2.3] or [61, p. 589]). In (4), for a fixed positive $n$, only finitely many terms are nonzero. In turn, Zeilberger [62] showed how to check to holonomicity and proved that a class of proper-hypergeometric functions is holonomic. Moreover, finite (multi-dimensional) sums of proper $q$-hypergeometric terms are $q$-holonomic in the remaining free variables. This is the Fundamental Theorem of Wilf and Zeilberger [61]. Hence, the terms $F$ in (4) are $q$-holonomic in all $r+1$ variables [12]. Recall that a discrete function $f \in \mathbb{Z}^{r} \rightarrow \mathbb{Q}(q)$ is $q$ hypergeometric iff $E_{i} f / f \in \mathbb{Q}\left(q, q^{n_{1}}, \ldots, q^{n_{r}}\right)$ for all $i=1, \ldots, r$. Here the operators $E_{i}, i=1, \ldots, r$, act on the discrete functions by $E_{i} f\left(n_{1}, \ldots, n_{r}\right)=f\left(n_{1}, \ldots, n_{i-1}, n_{i}+1, n_{i+1}, \ldots, n_{r}\right)$.

Remark. The asymptotics of solutions of $q$-difference equations at $q=\exp \{2 \pi i \alpha / n\}$ with some $\alpha \in[0,1]$ as $n \rightarrow \infty$ was studied by Garoufalidis and Geronimo [14] under a regular condition for Eq. (2). By definition, Eq. (2) is regular (see [14, Def. 1.1]) if

$$
\operatorname{Dsc}_{\lambda} P(v, \lambda) \cdot b_{0}(v, 1) \cdot b_{d}(v, 1) \neq 0 \text { for all } v \in \mathbb{S}^{1} \text {, }
$$


where $\operatorname{Dsc}_{\lambda} P(v, \lambda)$ is the discriminant of $P(v, \lambda)$. Another words, Eq. (2) is regular iff the eigenvalues of (2) (i.e., the roots of $P(v, \lambda)$ ) never collide and never vanish for every $v \in \mathbb{S}^{1}$.

The key role in the proof in [14] plays the translation of asymptotics of solutions of $q$-difference equations in the terms of asymptotics of solutions of $\varepsilon$-difference equations [14, Section 2]. In turn, the $\varepsilon$-difference equations of the form

$$
\sum_{j=0}^{l} a_{j}(k \varepsilon, \varepsilon) y_{k+j}=0, \quad k \in \mathbb{Z} \quad(l \text { fixed }),
$$

were considered by O. Costin and R. Costin [10]. Using the WKB method, they studied the asymptotics of solutions as $\varepsilon \rightarrow 0$.

In this paper we adopt for $q$-polynomials another approach for studying asymptotics of usual polynomials defined by the recurrence relations of high order (the approach was introduced and developed in $[2-6,55-57])$.

\section{VOLUME CONJECTURE}

In this section, we describe some conjectures concerning the colored Jones function.

\subsection{Hyperbolic Volume Conjecture}

Kashaev [28, 29] defined a complex-valued knot invariant $\langle K\rangle_{n}$ of a knot $K$ for $n=2,3, \ldots$ by using the quantum dilogarithm. He proposed a conjecture that his invariant $\langle K\rangle_{n}$ would grow exponentially w.r.t. $n$ and that its growth rate would give the volume of the complement of a hyperbolic knot:

$$
2 \pi \lim _{n \rightarrow \infty} \frac{1}{n} \log \left|\langle K\rangle_{n}\right|=\operatorname{vol}\left(\mathbb{S}^{3} \backslash K\right),
$$

where $\operatorname{vol}\left(\mathbb{S}^{3} \backslash K\right)$ is the volume of a complete hyperbolic metric in the knot complement $\mathbb{S}^{3} \backslash K$. Later, H. Murakami and J. Murakami [39, Theorem 4.9] proved that Kashaev's invariant with parameter $n$ coincides with $n$ dimensional colored Jones polynomial evaluated at the $n$-th root of unity,

$$
\langle K\rangle_{n}=\left.J_{K}^{\prime}(n, q)\right|_{q=\exp \{2 \pi i / n\}} \text {. }
$$

The value $\langle K\rangle_{n}$ is called the Kashaev invariant of a knot $K$ at $q=\exp \{2 \pi i / n\}$. For example, see [29], $\langle\text { unknot }\rangle_{n}=1$,

$$
\left\langle 3_{1}\right\rangle_{n}=\sum_{k=0}^{n-1}(q)_{k}, \quad\left\langle 4_{1}\right\rangle_{n}=\sum_{k=0}^{n-1}\left|(q)_{k}\right|^{2}, \quad\left\langle 5_{2}\right\rangle_{n}=\sum_{0 \leq k \leq l \leq n-1} \frac{(q)_{l}^{2}}{(\bar{q})_{k}} q^{-(l+1) k},
$$

where the $q$-factorial $(q)_{m}, m \in \mathbb{N}$, is defined by

$$
(q)_{m}=(1-q)\left(1-q^{2}\right) \cdot \ldots \cdot\left(1-q^{m}\right), \quad(q)_{0}:=1,
$$

and $\bar{q}$ denotes the complex conjugation of $q$.

The Hyperbolic Volume Conjecture (HVC, in short) states as follows.

(HVC): Let $K$ be a hyperbolic knot in $\mathbb{S}^{3}$. Then

$$
2 \pi \lim _{n \rightarrow \infty} \frac{\log \left|J_{K}^{\prime}\left(n, q=e^{2 \pi i / n}\right)\right|}{n}=\operatorname{vol}\left(\mathbb{S}^{3} \backslash K\right) .
$$

The (HVC) was generalized by H. Murakami and J. Murakami [39] to the any knot as follows.

Volume Conjecture: Let $K$ be any knot in $\mathbb{S}^{3}$. Then

$$
2 \pi \lim _{n \rightarrow \infty} \frac{\log \left|J_{K}^{\prime}\left(n, q=e^{2 \pi i / n}\right)\right|}{n}=v_{3}\left\|\mathbb{S}^{3} \backslash K\right\|,
$$

where $v_{3}$ is the volume of the ideal regular hyperbolic tetrahedron in 3-dimensional hyperbolic space $\mathbf{H}^{3}$ and $\|M\|$ is the Gromov norm [20] (or simplicial volume). In particular if the knot $K$ is hyperbolic, then the r.h.s. of (9) coincides with the volume of the knot complement $\operatorname{vol}\left(\mathbb{S}^{3} \backslash K\right)$. 
For details, see [39, Sec. 5] and the review book [40, Chapter 3].

Remarks. (i) The simplicial volume of $K,\left\|\mathbb{S}^{3} \backslash K\right\|$, is equal to the sum of the hyperbolic volumes of the hyperbolic pieces of the torus decomposition of $\mathbb{S}^{3} \backslash K$ divided by $v_{3}$.

(ii) Note that simplicial volumes of torus knots are known to be zero. For example, the trefoil knot (the $3_{1}$ knot in Rolfsen' notation [49]) is a torus knot [51]. Hence, $\left\|\mathbb{S}^{3} \backslash 3_{1}\right\|=0$. Kashaev and Tirkkonen [30] proved the Volume Conjecture for torus knots. They showed that in this case the l.h.s. of (8) vanishes, see also [16, Appendix B].

(iii) For the first time, the regular hyperbolic 3-simplex was considered by Gieseking in 1912 (see [19]). Consider a regular tetrahedron in Euclidean space, inscribed in the unit sphere, so that its vertices are on the sphere. Now interpret this tetrahedron to lie in the projective model for hyperbolic space, so that it determines an ideal hyperbolic simplex. The dihedral angles of the hyperbolic simplex are $\pi / 3$. The volume of regular ideal 3 -simplex is equal to $v_{3}=3 \Lambda(\pi / 3)=1.01494 \ldots$ (see [38]), where $\Lambda(z)$ is the Lobachevskii function,

$$
\Lambda(z)=-\int_{0}^{z} \log |2 \sin u| d u .
$$

Note that the Lobachevskii function can be expressed by the imaginary part of the Euler dilogarithm $\operatorname{Li}_{2}(z)$ [29]. For $z \in \mathbb{C}$ such that $|z| \leq 1$, the Euler dilogarithm is defined by

$$
\mathrm{Li}_{2}(z):=\sum_{r=1}^{\infty} \frac{z^{r}}{r^{2}} .
$$

For $z \in \mathbb{C}$ such that $|\arg (z)|<\pi$, the analytic continuation of the dilogarithm function is given by

$$
\operatorname{Li}_{2}(z)=-\int_{0}^{z} \frac{\log (1-u)}{u} d u
$$

with $0<\arg (1-z)<2 \pi$. Then, for $\theta \in \mathbb{R}$, the Lobachevskii function is defined by the rule

$$
\Lambda(\theta)=\frac{1}{2} \Im\left(\operatorname{Li}_{2}(\exp (2 \theta i))\right)=\frac{1}{2} \sum_{r=1}^{\infty} \frac{\sin (2 r \theta)}{r^{2}} .
$$

(iv) In 1975, R. Riley [48] showed that the figure-eight knot complement has a hyperbolic structure. Thus, the figure-eight knot (the $4_{1}$ knot in Rolfsen' notation [49]) is a hyperbolic knot. In 1978, Thurston proved that the figure-eight complement can be triangulated by two copies of a maximal 3simplex. Hence, $\operatorname{vol}\left(\mathbb{S}^{3} \backslash 4_{1}\right)=2 v_{3}=6 \Lambda(\pi / 3)=2.02988 \ldots$ This formula was obtained by Milnor [38]. Similarly, the complement of the Whitehead link was constructed from a regular ideal octahedron which in turn, is formed by gluing two copies of the infinite cone on a regular planar quadrilateral. Thus, its volume equals $8 \Lambda(\pi / 4)=3.66386 \ldots$. The complement of the Borromean rings has volume $16 \Lambda(\pi / 4)=7.32772 \ldots$, since it is obtained by gluing two ideal octahedra together, see Example in [51, Sect. 7.2].

(v) There are examples of knots all with the same volume. For example, the $5_{2}$ knot and a 12 -crossing knot $12 n \_242$ have the same volume, $\operatorname{vol}\left(\mathbb{S}^{3} \backslash 5_{2}\right)=\operatorname{vol}\left(\mathbb{S}^{3} \backslash 12 n \_242\right)=2,82812 \ldots$ (cf. [36]). There are other invariants which can be used to distinguish between manifolds of the same volume, for example, the maximal cusp volume [1, Sections 3, 4].

(vi) The volume conjecture was confirmed for $4_{1}$ by Ekholm (see, e.g., [40]); $5_{2}$ by Kashaev [29] and Ohtsuki [41]; $6_{1}$ by Kashaev [29]; Whitehead chains by van der Veen [60]; twisted Whitehead links by Zheng [63]; Borromean rings by Garoufalidis and Lê [15]; for knots with up to 7 crossings by Ohtsuki, Yokota [42, 43].

To numerically confirm the volume conjecture, the authors use the explicit multisum formulas for the Kashaev invariant (cf. (7)). These formulas allow to reduce the finding the limit in the 1.h.s. of (9) to the study of the asymptotics of special functions such that the Lobachevskii function $\Lambda(z)$, the Euler 
dilogarithm $\mathrm{Li}_{2}(z)$, with applying the saddle point method. Namely, the first step in this approach is to write the Kashaev invariant $\langle K\rangle_{N}$ in the form

$$
\langle K\rangle_{N} \sim \int \exp \left(\frac{i N}{2 \pi} V_{K}(z)\right) d z_{1} \ldots d z_{p} \text { for large } N
$$

where $p$ is the number of summations appearing in $\langle K\rangle_{N}$. The function $V_{K}(z), z \in \mathbb{C}^{p}$, is called a potential. To find this function, one uses the following asymptotic behavior

$$
q^{k l} \sim \exp \left(-\frac{i N}{2 \pi} \log q^{k} \log q^{l}\right), \quad(q)_{n} \sim \exp \left(\frac{i N}{2 \pi}\left(\operatorname{Li}_{2}\left(q^{n}\right)-\frac{\pi^{2}}{6}\right)\right) .
$$

The second step is to find a saddle point $z_{0}$ which is a solution to $\left.\frac{\partial}{\partial z_{j}} V_{K}(z)\right|_{z=z_{0}}=0$ for any $j=$ $1, \ldots, p$. Then, $\langle K\rangle_{N} \sim \exp \left(\frac{i N}{2 \pi} V_{K}\left(z_{0}\right)\right)$. For example, for the $4_{1}$ knot, the potential is $V_{4_{1}}(z)=$ $\mathrm{Li}_{2}(z)-\mathrm{Li}_{2}\left(z^{-1}\right)$, the saddle point is $z_{0}=\exp (-\pi i / 3)$ (see [24, p. 333]).

\subsection{AJ Conjecture}

The next AJ conjecture (made by Garoufalidis [13, p. 297]) relates the colored Jones polynomial and the $A$-polynomial. ( $A J$ are the initials of the $A$-polynomial and the colored Jones polynomial $J$ ). The $A$ polynomial of a knot was introduced in [11]. It is a two-variable polynomial, usually written in the terms of the meridian and longitude variables $M$ and $L$. The $A$-polynomial, $A_{K}(M, L)$, parameterizes the affine variety of $\operatorname{SL}(2, \mathbb{C})$ representations of the knot complement, viewed from the boundary torus [11]. The $A$-polynomial is defined up to multiplication by a rational function of $M$ and a power of $L$ [13].

Since the colored Jones polynomial is defined by a multidimensional sum of a proper $q$-hypergeometric term, numerous algorithms can produce a linear recurrence with polynomial coefficients; see, e.g., the book of Petkovšek, Wilf and Zeilberger [46]. Different algorithms in general produce different recurrences, which may not be of minimal order. The non-commutative $A$-polynomial of the twist knots $K_{p}$ was computed with a certificate by Garoufalidis and Sun [18] for $p=-15 ; \ldots ; 15$. This polynomial was calculated for most knots up to 12 crossings, see $[11,36]$. To state the $A J$ conjecture we write $q$-difference equations in the operator form

$$
P f=0, \quad \text { where } P:=\sum_{j=0}^{d} b_{j}(Q, q) E^{j} .
$$

The operators $E, Q$, and $q$ act on a discrete function $f: \mathbb{N} \rightarrow \mathbb{Z}\left[q^{ \pm 1}\right]$ by the rule

$$
(q f)(n)=q f(n), \quad(Q f)(n)=q^{n} f(n), \quad(E f)(n)=f(n+1) .
$$

Then, we put $q=t^{4},(L f)(n):=f(n+1),(M f)(n):=t^{2 n} f(n)$. Then $J_{K}^{\prime}(n)$ satisfies the following equation

$$
\alpha_{k}(f)=0, \quad \alpha_{k} \equiv \alpha_{K}(t ; M, L):=\sum_{j=0}^{d} \alpha_{K, j}(t ; M) L^{j} .
$$

The AJ conjecture (cf. [13]): For every knot $K,\left.\alpha_{K}\right|_{t= \pm 1}$ is $M$-essentially equal to the A-polynomial (i.e., $\left.\alpha_{K}\right|_{t= \pm 1}$ is equal to the A-polynomial of the knot $K$ up to a factor depending on $M$ only). Note that $\alpha_{K}$ is defined up to a factor $\pm t^{a} M^{b}, a, b \in \mathbb{Z}$. Another form of this conjecture is the characteristic polynomial

$$
\operatorname{ch} P_{K}(\lambda, v)=\left.A_{K}(L, M)\right|_{\left(L, M^{2}\right)=(\lambda, v)}
$$

up to a multiplication by a polynomial in $v$. For example, for $K=3_{1}$,

$$
\left.\alpha_{K}\right|_{t=-1}=\left(M^{4}-1\right)(L-1)\left(L M^{6}+1\right)=\left(M^{4}-1\right) A_{K}(L, M),
$$

where $A_{K}(L, M)$ is $A$-polynomial for $K=3_{1}$. Cooper et al [11] computed the $A$-polynomial of the $3_{1}$ and $4_{1}$ knots (see also [13, p. 300])

$$
A_{3_{1}}(L, M)=(L-1)\left(L M^{6}+1\right), \quad A_{-3_{1}}(L, M)=(L-1)\left(L+M^{6}\right),
$$



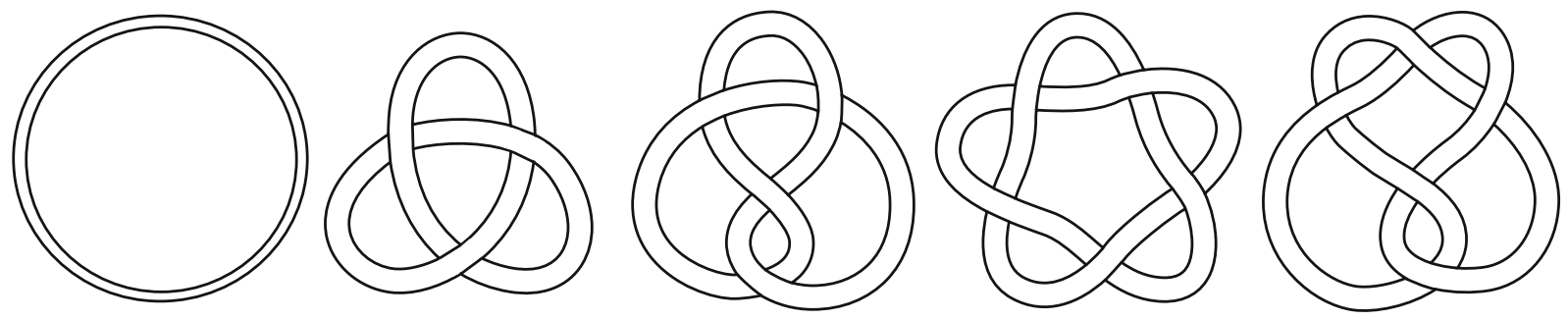

Fig. 1. The unknot and the $3_{1}, 4_{1}, 5_{1}$ and $5_{2}$ knots.

$$
A_{4_{1}}(L, M)=(L-1)\left(-L+L M^{2}+M^{4}+2 L M^{4}+L^{2} M^{4}+L M^{6}-L M^{8}\right),
$$

where the $-3_{1}$ knot is the mirror image of the $3_{1}$ knot. The $A$-polynomial of the $5_{2}$ knot is given by (cf. $[36,11])$

$$
\begin{aligned}
A_{5_{2}}(M, L)= & L^{3} M^{14}+L^{2}\left(-M^{14}+2 M^{12}+2 M^{10}-M^{6}+M^{4}\right) \\
& +L\left(M^{10}-M^{8}+2 M^{4}+2 M^{2}-1\right)+1 .
\end{aligned}
$$

Correspondingly (we change $L$ into $L^{-1}$ and omit the factor $L^{-3}$ ),

$$
\begin{gathered}
A_{-5_{2}}(M, L)=L^{3}+L^{2}\left(M^{10}-M^{8}+2 M^{4}+2 M^{2}-1\right) \\
\quad+L\left(-M^{14}+2 M^{12}+2 M^{10}-M^{6}+M^{4}\right)+M^{14}
\end{gathered}
$$

The $A J$ conjecture was confirmed for the knots $33_{1}, 4_{1}, 7_{4}$ (by Garoufalidis [13] and KoutschanGaroufalidis [17]); for torus knots (by Hikami [25], Tran [54]), for some classes of two-bridge knots, including all twist knots, and pretzel knots (by Le [33], Le-Tran [34], Le-Zhang [35]).

Remark. There are new conjectures about the colored Jones polynomial, for details, see, e.g., [53].

\section{THE COLORED JONES FUNCTION}

Habiro [23] obtained the following expansion of the colored Jones function, known as the cyclotomic expansion. He showed that for every zero-framed knot $K$, there exists a cyclotomic function $C_{K}$ : $\mathbb{Z}_{>0} \rightarrow \mathbb{Z}\left[q^{ \pm 1}\right]$ such that

$$
J_{K}^{\prime}(n)=\sum_{k=0}^{n-1} C_{K}(k) s(n, k), \quad n \in \mathbb{N},
$$

where $C_{K}(0):=1$, the cyclotomic kernel $s(n, k)$ is a proper $q$-hypergeometric term given by (see e.g., $[13$, p. 300; 18, p. 1576])

$$
\begin{aligned}
s(n, k) & =\frac{\{n-k\}\{n-k+1\} \cdots\{n+k-1\}\{n+k\}}{\{n\}} \\
& =q^{n k} \prod_{j=1}^{k}\left(1-q^{j-n}\right)\left(1-q^{-j-n}\right)=\prod_{j=1}^{k}\left(q^{n}+q^{-n}-q^{j}-q^{-j}\right), \quad \text { with } s(n, 0):=1 .
\end{aligned}
$$

Here $\{n\}:=q^{n / 2}-q^{-n / 2}$. Masbaum [37] obtained a formula for the colored Jones polynomial of twist knots generalizing formula of Habiro and Lê [32] for the $3_{1}$ and $4_{1}$ knots. Note that Garoufalidis and Lê [12] proved that the function $C_{K}$ of every knot $K$ is $q$-holonomic. For the unknot, the function $C_{\text {unknot }}(k)=\delta_{k 0}$, where $\delta_{k 0}$ denotes the Kronecker delta. Below we give examples of the function $C_{K}$ and the colored Jones function $J_{K}^{\prime}$ for the $3_{1}, 4_{1}$ and $5_{2}$ knots. The unknot and the first four knots are shown in Fig. 1. The pictures for the next knots see in [49].

Examples. 1) The trefoil knot is the simplest non-trivial knot. The (right-handed) $3_{1}$ knot has the Jones polynomial of the form $J_{3_{1}}^{\prime}(q)=q^{-1}+q^{-3}-q^{-4}$ (cf. [36]). The $3_{1}$ knot is chiral (i.e., the knot that is not equivalent to its mirror image). For the (left-handed) trefoil $\left(-3_{1}\right)$, the mirror image of $3_{1}$,

$$
J_{-3_{1}}^{\prime}(q)=q+q^{3}-q^{4} .
$$


The cyclotomic function $C_{K}$ for the right-handed trefoil knot is equal to $C_{3_{1}}(k)=(-1)^{k} q^{-k(k+3) / 2}$ (cf. [37, p. 555; 13, p. 299]). For the left-handed trefoil, $J_{-3_{1}}^{\prime}(n, q)=J_{3_{1}}^{\prime}\left(n, q^{-1}\right)$, hence, by (16),

$$
J_{-3_{1}}^{\prime}(n, q)=\sum_{k=0}^{n-1}(-1)^{k} q^{k(k+3) / 2} s(n, k) .
$$

2) The figure-eight knot is the simplest hyperbolic knot. This knot has Jones polynomial of the form $J_{4_{1}}^{\prime}(q)=q^{2}-q+1-q^{-1}+q^{-2}$ (cf. [36]). The $4_{1}$ knot is achiral. For this knot, the function $C_{K}$ equals $C_{4_{1}}(k)=1$. Hence, by (16), the colored Jones function is $J_{4_{1}}^{\prime}(n, q)=\sum_{k=0}^{n-1} s(n, k)$. In particular, the $N$ colored Jones polynomial $J_{K}^{\prime}(N ; q)$ evaluated at $q=\exp \{2 \pi i / N\}$ is

$$
J_{4_{1}}^{\prime}(N, q=\exp \{2 \pi i / N\})=\left.\sum_{k=0}^{N-1} s(N, k)\right|_{q=\exp \{2 \pi i / N\}}=\sum_{k=0}^{N-1}(q)_{k}\left(q^{-1}\right)_{k}=\sum_{k=0}^{N-1}\left|(q)_{k}\right|^{2},
$$

what coincides with [29, formula (2.2)].

3) The Jones polynomial of the $5_{2}$ knot is (cf. [36])

$$
J_{5_{2}}^{\prime}(q)=-q^{-6}+q^{-5}-q^{-4}+2 q^{-3}-q^{-2}+q^{-1} .
$$

The $5_{2}$ knot is chiral, the Jones polynomial of the mirror of $5_{2}$ is $J_{-5_{2}}^{\prime}(q)=J_{5_{2}}^{\prime}\left(q^{-1}\right)$ and $C_{-5_{2}}(k, q)=$ $C_{5_{2}}\left(k, q^{-1}\right)$. For this knot, the cyclotomic function equals (cf. [37, Theorem 5.1])

$$
C_{5_{2}}(k) \equiv C_{5_{2}}(k, q)=(-1)^{k} q^{-\frac{3 k^{2}+5 k}{2}} \sum_{m=0}^{k} q^{-\frac{m^{2}-2 m-3 k m}{2}} \frac{[k] !}{[m] ![k-m] !},
$$

where $[k] !=[1][2] \cdots[k],[0] !:=1,[k]$ is defined in (1). In particular, (19) implies

$$
\begin{gathered}
C_{5_{2}}(0)=1, \quad C_{5_{2}}(1)=-q^{-4}-q^{-2}, \quad C_{5_{2}}(2)=q^{-11}+q^{-8}+q^{-7}+q^{-5}, \\
C_{5_{2}}(3)=q^{-21}+q^{-17}+q^{-16}+q^{-15}+q^{-13}+q^{-12}+q^{-11}+q^{-9} .
\end{gathered}
$$

For the $-5_{2}$ knot, $C_{-5_{2}}(k, q)=C_{5_{2}}\left(k, q^{-1}\right)$. The colored Jones function for the $5_{2}$ knot is given by (16), (17), and (19). In particular, using (16), (17), and (20), we calculate the colored Jones function $J_{5_{2}}^{\prime}(n, q)$ for $n=1,2,3$

$$
\begin{aligned}
& \left.J_{5_{2}}^{\prime}(n, q)\right|_{n=1}=1 \\
& \left.J_{5_{2}}^{\prime}(n, q)\right|_{n=2}=-q^{-6}+q^{-5}-q^{-4}+2 q^{-3}-q^{-2}+q^{-1} \\
& \left.J_{5_{2}}^{\prime}(n, q)\right|_{n=3}=q^{-17}-q^{-16}-q^{-15}+2 q^{-14}-q^{-13}-2 q^{-12}+3 q^{-11}-q^{-10} \\
& \quad-3 q^{-9}+4 q^{-8}-q^{-7}-2 q^{-6}+3 q^{-5}-q^{-3}+q^{-2}
\end{aligned}
$$

\section{4. $q$-DIFFERENCE EQUATIONS}

The recurrence relations for the colored Jones function can be found from multisum-formulas for the colored Jones function of a form (16) by computer calculations. For details, see [13, Chapter 3.2]. There are various programs that can compute the recursion relations for multisums, see, e.g., $[18,45,46]$ and the references therein. Below we give $q$-difference equations in the case of the $3_{1}$ and $4_{1}$ knots. For the $5_{2}$ knot, the $q$-difference equation is given in Section 5.

\section{1. q-Difference Equation for the Trefoil Knot}

For $K=-3_{1}$, on the first step the program gives a recurrence relation for the (left-handed) trefoil $J_{-3_{1}}^{\prime}(n, q)$ of a form

$$
J_{-3_{1}}^{\prime}(n)=q^{-2+n} \frac{-q+q^{2 n}}{-1+q^{n}}-q^{-1+3 n} \frac{1-q^{-1+n}}{1-q^{n}} J_{-3_{1}}^{\prime}(n-1) .
$$


Put $J_{-3_{1}}^{\prime}(0)=0$. Then $J_{-3_{1}}^{\prime}(1)=1$ and $J_{-3_{1}}^{\prime}(2)=q+q^{3}-q^{4}$ (cf. formula (18)). Relation (22) is a first order inhomogeneous recurrence relation. On the second step, the program converts (22) into a second order homogeneous recurrence relation

$$
\sum_{j=0}^{2} b_{j}\left(q^{n}, q\right) J_{-3_{1}}^{\prime}(n+j)=0
$$

where the coefficients $b_{j}, j=0,1,2$, are

$$
\begin{aligned}
b_{0}=\frac{q^{2 n+2}\left(q^{n}-1\right)}{\left(q^{2 n+1}-1\right)}, \quad b_{1} & =\frac{\left(q^{n+1}-1\right)\left(-1+q^{2 n+3}+q^{3 n+4}-q^{5 n+5}\right)}{q^{n}\left(q^{2 n+3}-1\right)\left(q^{2 n+1}-1\right)}, \\
b_{2} & =\frac{q^{n+2}-1}{q^{n+1}\left(1-q^{2 n+3}\right)} .
\end{aligned}
$$

The initial conditions for $(23)$ are $J_{-3_{1}}^{\prime}(0)=0, J_{-3_{1}}^{\prime}(1)=1$. The characteristic polynomial for this knot is of the form

$$
P_{-3_{1}}(v, \lambda)=\sum_{j=0}^{2} b_{j}(v, 1) \lambda^{j}=-\frac{(\lambda-1)\left(\lambda+v^{3}\right)}{v(1+v)}, \quad \operatorname{Dsc}_{\lambda} P_{-3_{1}}(v, \lambda)=\frac{\left(1-v+v^{2}\right)^{2}}{v^{2}} .
$$

The eigenvalues are $\lambda_{1}=1$ and $\lambda_{2}=-v^{3}$. In particular, $\operatorname{Dsc}_{\lambda} P_{-3_{1}}(v, \lambda)=0$ for $v_{1,2}=e^{ \pm \pi i / 3} \in \mathbb{S}^{1}$. Therefore, the regularity condition (5) does not hold.

\section{2. q-Difference Equation for the Figure-Eight Knot}

For the $4_{1}$ knot, on the first step we obtain a second-order inhomogeneous recurrence relation of the form (cf. [13, p. 303])

$$
\begin{gathered}
J_{4_{1}}^{\prime}(n)=\frac{q^{1-n}\left(1+q^{n-1}\right)\left(1-q^{2 n-1}\right)}{1-q^{n}}-\frac{\left(1-q^{n-2}\right)\left(1-q^{2 n-1}\right)}{\left(1-q^{n}\right)\left(1-q^{2 n-3}\right)} J_{4_{1}}^{\prime}(n-2) \\
+\frac{q^{2-2 n}\left(1-q^{n-1}\right)^{2}\left(1+q^{n-1}\right)\left(1+q^{4 n-4}-q^{n-1}-q^{2 n-3}-q^{2 n-1}-q^{3 n-3}\right)}{\left(1-q^{n}\right)\left(1-q^{2 n-3}\right)} J_{4_{1}}^{\prime}(n-1),
\end{gathered}
$$

which is converted into a third-order homogeneous recurrence relation of the form (after change $n \rightarrow$ $n+3)$

$$
\sum_{j=0}^{3} b_{j}\left(q^{n}, q\right) J_{4_{1}}^{\prime}(n+j)=0
$$

where the coefficients $b_{j} \equiv b_{j}\left(q^{n}, q\right), j=0,1,2,3$, are

$$
b_{0}=\frac{q^{n+1}\left(q^{n}-1\right)}{\left(1+q^{n+1}\right)\left(q^{2 n+1}-1\right)}, \quad b_{3}=\frac{q^{n+2}\left(q^{n+3}-1\right)}{\left(1+q^{n+2}\right)\left(1-q^{2 n+5}\right)}
$$

and

$$
\begin{aligned}
& b_{1}=\frac{\left(q^{n+1}-1\right)\left(1-2 q^{n+1}+q^{n+2}-q^{2 n+1}+q^{2 n+2}-q^{2 n+3}+q^{3 n+2}-2 q^{3 n+3}+q^{4 n+4}\right)}{q^{n+1}\left(1+q^{n+2}\right)\left(1-q^{2 n+1}\right)}, \\
& b_{2}=\frac{\left(q^{n+2}-1\right)\left(1+q^{n+1}-2 q^{n+2}-q^{2 n+3}+q^{2 n+4}-q^{2 n+5}-2 q^{3 n+6}+q^{3 n+7}+q^{4 n+8}\right)}{q^{n+2}\left(1+q^{n+1}\right)\left(q^{2 n+5}-1\right)} .
\end{aligned}
$$

Remark. Note that the coefficients $b_{j}$ in $(24)$ and $(27)$ have the factor $\left(q^{n+j}-1\right)$ for all $j$. This fact is valid for any knot and follows from (16) and the explicit formula (17) for the kernel $s(n, k)$ since

$$
s(n, k)=\frac{\gamma(n, k)}{1-q^{n}}, \quad \gamma(n, k):=\prod_{j=n-k}^{n+k}\left(1-q^{j}\right) .
$$


The initial conditions for $(25)$ are $J_{4_{1}}^{\prime}(0)=0, J_{4_{1}}^{\prime}(1)=1, J_{4_{1}}^{\prime}(2)=q^{2}-q+1-q^{-1}+q^{-2}$.

In this case, the characteristic polynomial is

$$
P_{4_{1}}(v, \lambda)=\sum_{j=0}^{3} b_{j}(v, 1) \lambda^{j}=-\frac{(\lambda-1)\left(v^{2} \lambda^{2}-\lambda\left(1-v-2 v^{2}-v^{3}+v^{4}\right)+v^{2}\right)}{v(1+v)^{2}} .
$$

Hence, for the $4_{1}$ knot, we have 3 eigenvalues, $\lambda_{1}(v), \lambda_{2}(v)=1 / \lambda_{1}(v), \lambda_{3}=1$, where

$$
\lambda_{1,2}=\frac{1}{2 v^{2}}\left(1-v-2 v^{2}-v^{3}+v^{4} \pm \sqrt{D}\right),
$$

$D:=\left(1-v-2 v^{2}-v^{3}+v^{4}\right)^{2}-4 v^{4}=(1+v)^{2}(1-v)^{2}\left(1+v+v^{2}\right)\left(1-3 v+v^{2}\right)$. Hence, $\lambda_{1}=\lambda_{2}$ for $v= \pm 1, e^{2 \pi i / 3}, e^{4 \pi i / 3} \in \mathbb{S}^{1}$. Thus, the regularity condition does not hold.

\section{A THIRD-ORDER $q$-DIFFERENCE EQUATION}

Consider a third-order inhomogeneous $q$-difference equation of the form

$$
\sum_{j=0}^{3} A_{j}\left(q^{n}, q\right) f_{n+j}(q)=B\left(q^{n}, q\right)
$$

where $A_{j} \equiv A_{j}\left(q^{n}, q\right), B \equiv B\left(q^{n}, q\right)$ are

$$
\begin{aligned}
B= & q^{2 n+4}\left(q^{n+1}+1\right)\left(q^{n+2}+1\right)\left(q^{2 n+1}-1\right)\left(q^{2 n+3}-1\right)\left(q^{2 n+5}-1\right), \\
A_{0}= & q^{7 n+9}\left(q^{2 n+4}-1\right)\left(q^{2 n+5}-1\right), \\
A_{1}= & q^{2 n+5}\left(q^{2 n+2}-1\right)\left(q^{2 n+5}-1\right)\left(1-q^{n+1}-q^{2 n+1}+q^{2 n+2}+q^{2 n+3}-q^{2 n+4}\right. \\
& \left.\quad+q^{3 n+2}+q^{3 n+5}+2 q^{4 n+5}-q^{5 n+6}\right), \\
A_{2}= & q\left(q^{2 n+4}-1\right)\left(q^{2 n+1}-1\right)\left(-1+2 q^{n+2}+q^{2 n+2}+q^{2 n+5}-q^{3 n+4}+q^{3 n+5}\right. \\
& \left.+q^{3 n+6}-q^{3 n+7}-q^{4 n+7}+q^{5 n+9}\right), \\
A_{3}= & \left(q^{2 n+2}-1\right)\left(q^{2 n+1}-1\right) .
\end{aligned}
$$

The initial conditions for (28) are

$$
\begin{aligned}
\left.f_{n}\right|_{n=1}= & q-1, \\
\left.f_{n}\right|_{n=2}= & \left(q^{2}-1\right)\left(-q^{6}+q^{5}-q^{4}+2 q^{3}-q^{2}+q\right), \\
\left.f_{n}\right|_{n=3}=\left(q^{3}-1\right)\left(q^{17}-q^{16}-q^{15}+2 q^{14}-q^{13}-2 q^{12}+3 q^{11}-q^{10}-3 q^{9}+4 q^{8}-q^{7}\right. & \left.\quad-2 q^{6}+3 q^{5}-q^{3}+q^{2}\right) .
\end{aligned}
$$

Lemma 1. The solution to problem (28), (30) is $f_{n}(q)=\left(q^{n}-1\right) J_{-5_{2}}^{\prime}(n, q)$, where $J_{-5_{2}}^{\prime}(n, q)=$ $J_{5_{2}}^{\prime}\left(n, q^{-1}\right)$ and the colored Jones function for the $5_{2}$ knot is given by (16), (17), and (19).

Proof. At first, we find the corresponding to (28) fourth-order homogeneous $q$-difference equation. Indeed, the solution of (28) satisfies the following equation

$$
\sum_{j=0}^{4} b_{j}\left(q^{n}, q\right) f_{n+j}(q)=0
$$

where the coefficients $b_{j}:=b_{j}\left(q^{n}, q\right)$ are

$$
\begin{aligned}
& b_{0}=\frac{q^{5 n+5}\left(q^{n+2}-1\right)}{\left(q^{n+1}+1\right)\left(q^{2 n+1}-1\right)\left(q^{2 n+3}-1\right)}, \quad b_{4}=-\frac{q^{n+2}-1}{q^{2 n+6}\left(q^{n+3}+1\right)\left(q^{2 n+5}-1\right)\left(q^{2 n+7}-1\right)}, \\
& b_{1}=\frac{q\left(q^{n+1}-1\right)\left(1-q^{n+1}-(q-1)\left(q^{2}-1\right) q^{2 n+1}+\left(q^{3}+1\right) q^{3 n+2}+2 q^{4 n+5}-q^{5 n+6}\right)}{\left(q^{n+2}+1\right)\left(q^{2 n+1}-1\right)\left(q^{2 n+3}-1\right)}
\end{aligned}
$$




$$
\begin{gathered}
-\frac{q^{5 n+10}\left(q^{n+3}-1\right)}{\left(q^{n+2}+1\right)\left(q^{2 n+3}-1\right)\left(q^{2 n+5}-1\right)}, \\
b_{2}=\frac{\left(q^{n+2}-1\right)\left(q^{5 n+9}-q^{4 n+7}-(q-1)\left(q^{2}-1\right) q^{3 n+4}+\left(q^{3}+1\right) q^{2 n+2}+2 q^{n+2}-1\right)}{\left(q^{2 n+3}-1\right)\left(q^{2 n+5}-1\right) q^{2 n+3}\left(q^{n+1}+1\right)} \\
-\frac{q\left(q^{n+2}-1\right)\left(1-q^{n+2}-(q-1)\left(q^{2}-1\right) q^{2 n+3}+\left(q^{3}+1\right) q^{3 n+5}+2 q^{4 n+9}-q^{5 n+11}\right)}{\left(q^{2 n+3}-1\right)\left(q^{2 n+5}-1\right)\left(q^{n+3}+1\right)}, \\
b_{3}=\frac{1}{q^{2 n+4}\left(q^{n+2}+1\right)\left(q^{2 n+5}-1\right)}\left[\frac{q^{n+1}-1}{q^{2 n+3}-1}\right. \\
\left.-\frac{\left(q^{n+3}-1\right)\left(q^{5 n+14}-q^{4 n+11}-(q-1)\left(q^{2}-1\right) q^{3 n+7}+\left(q^{3}+1\right) q^{2 n+4}+2 q^{n+3}-1\right)}{q\left(q^{2 n+7}-1\right)}\right] .
\end{gathered}
$$

Secondly, the system (31) can be rewritten in the operator form

$$
P(q, Q, E) f \equiv\left(\sum_{j=0}^{4} b_{j}(Q, q) E^{j}\right) f=0
$$

where the operators $q, Q$ and $E$ are defined in (12). Further, let us put (cf. (13))

$$
\alpha(t, M, L):=\left.P(q, Q, E)\right|_{q=t^{4}, L=E, Q=M^{2}} .
$$

Using (32), we obtain ( see AJ conjecture in Section 2.2)

$$
\left.\alpha(t, M, L)\right|_{t=-1}=\frac{1-L}{\left(M^{2}+1\right)^{2}\left(M^{4}-1\right) M^{4}} A_{-5_{2}}(M, L),
$$

where $A_{-5_{2}}(M, L)$ is defined in (15). Using $A_{j}$ defined in (29) we write

$$
\tilde{A}_{j}\left(q^{n}, q\right):=\left(q^{n+j}-1\right) A_{j}\left(q^{n}, q\right), \quad j=0,1,2,3 .
$$

Then, the $q$-difference equation for the colored Jones function of the $-5_{2}$ knot is (cf. [18, p. 1575])

$$
\sum_{j=0}^{3} \tilde{A}_{j}\left(q^{n}, q\right) J_{-5_{2}}^{\prime}(n+j, q)=B\left(q^{n}, q\right) .
$$

Finally, the initial conditions for (28) are (cf. (21)) $\left.f_{n}\right|_{n=k}=\left.\left(q^{n}-1\right) J_{-5_{2}}^{\prime}(n, q)\right|_{n=k}, k=1,2,3$.

Remark. We note that the regularity condition does not hold for the $q$-recurrences (31)-(32). Indeed, put $q^{n}=v$ and $q=1$ in the coefficients $b_{j}\left(q^{n}, q\right)$ and obtain

$$
\begin{gathered}
b_{0}(v, 1)=\frac{v^{5}}{(v+1)^{3}(v-1)}, \quad b_{1}(v, 1)=\frac{1-v+2 v^{3}+2 v^{4}-2 v^{5}}{(v+1)^{3}(v-1)}, \\
b_{2}(v, 1)=\frac{v^{6}-v^{5}-2 v^{4}-3 v^{3}-2 v^{2}-v+1}{v^{2}(v+1)^{3}}, \\
b_{3}(v, 1)=\frac{-v^{5}+v^{4}-2 v^{2}-2 v+2}{v^{2}(v+1)^{3}(v-1)}, \quad b_{4}(v, 1)=-\frac{1}{v^{2}(v+1)^{3}(v-1)} .
\end{gathered}
$$

The characteristic polynomial for $(31)$ is

$$
P(v, \lambda)=\sum_{j=0}^{4} b_{j}(v, 1) \lambda^{j}=-\frac{(\lambda-1)\left(\lambda^{3}+\lambda^{2} a_{2}(v)+\lambda a_{1}(v)+v^{7}\right)}{v^{2}(v+1)^{3}(v-1)},
$$

where

$$
\begin{aligned}
& a_{2}(v):=v^{5}-v^{4}+2 v^{2}+2 v-1, \\
& a_{1}(v):=-v^{7}+2 v^{6}+2 v^{5}-v^{3}+v^{2}=-v^{2}\left(v^{5}-2 v^{4}-2 v^{3}+v-1\right) .
\end{aligned}
$$

It can be easily checked (details see below) that there exist points $v \in \mathbb{S}^{1} \operatorname{such}_{\text {that }} \operatorname{Dsc}_{\lambda} P(v, \lambda)=0$. 
The $q$-recurrence relations (28)-(30) have been presented to us by Stavros Garoufalidis ${ }^{1)}$. Being motivated by the Volume Conjecture to develop technique for asymptotic analysis of the solutions of $q$-difference equations, we have started in preprint [5] to investigate the asymptotics of the polynomials $\dot{J}_{n}(q):=f_{n}(q)$ to problem $(28)-(30)$ in a double scale as

$$
q \rightarrow 1, \quad n \rightarrow \infty, \quad q^{n}=z \in \mathcal{K} \Subset \overline{\mathbb{C}} .
$$

Here we report on some first steps in this direction.

\subsection{Homogeneous Problem. New Variables}

To find the asymptotics of a sequence of polynomials defined by inhomogeneous recurrences, we first construct an asymptotic expansion basis of solutions to the homogeneous problem

$$
\vec{Q}_{n+1}(q)=\mathcal{A}_{n} \vec{Q}_{n}(q), \quad \vec{Q}_{n}:=\left(Q_{n}, Q_{n+1}, Q_{n+2}\right)^{T},
$$

where the elements of the transition matrix

$$
\mathcal{A}_{n}=\left(\begin{array}{ccc}
0 & 1 & 0 \\
0 & 0 & 1 \\
\frac{A_{0}}{A_{3}} & \frac{A_{1}}{A_{3}} & \frac{A_{2}}{A_{3}}
\end{array}\right)
$$

are given in (29).

Instead of the basic variables $q$ and $n$, we will consider the following parameters. We will distinguish between large $N$ and current $n$ :

$$
N \rightarrow \infty, \quad \frac{n}{N}=t \in[0,1]
$$

Next, recall the previously introduced variable $z:=q^{n}$. Finally, define a parameter $H$ such that

$$
H:=N \ln q, \quad \text { i.e., } \quad q^{N}=: \boldsymbol{e}^{H} \Rightarrow\left\{\begin{array}{l}
q^{n}=z=\boldsymbol{e}^{t H} \\
q=\exp \left(\frac{H}{N}\right)=1+\frac{H}{N}+\ldots,
\end{array}\right.
$$

As a result, instead of the variables $n, q$ and $q^{n}$, the basic variables will be

$$
\left(n, q, q^{n}\right) \quad \longrightarrow \quad(t N, H / N, z)
$$

In view of (8) we have $H=2 \pi i$. The small parameter for the power expansions will be $(1 / N)$.

\subsection{Spectral Curve of the Homogeneous Problem. Branch Points}

Substituting into the characteristic equation

$$
\operatorname{det}\left(\mathcal{A}_{n}-I \lambda\right)=0
$$

the limit values $q^{n}=: z, q=: 1$, we obtain the equation

$$
\lambda(z): \quad \lambda^{3}+a_{2}(z) \lambda^{2}+a_{1}(z) \lambda+z^{7}=0
$$

for the limiting spectral curve, where $a_{1}$ and $a_{2}$ are defined in (36).

The genus of the algebraic function $\lambda(z)$ is 2 . It has eight branch points, two of them lie on the unit circle centered at origin

$$
z_{1,2}=\frac{1}{4}(3-\sqrt{13+16 \sqrt{2}} \pm i \sqrt{-6+6 \sqrt{13+16 \sqrt{2}}-16 \sqrt{2}}),
$$

their numerical values are equal to $z_{1,2}=-0.7422176660 \pm i 0.6701588892$.

Note also that although the points $z= \pm 1$ are not branch points, however at these points all three branches of the function $\lambda(z)=\left\{\lambda_{j}(z)\right\}_{j=0}^{2}$ coincide:

$$
\lambda_{0}( \pm 1)=\lambda_{1}( \pm 1)=\lambda_{2}( \pm 1) .
$$

\footnotetext{
${ }^{1)}$ Private communication.
} 


\subsection{Road Map for the Asymptotics}

Recall that our goal is to find the asymptotics (8)

$$
\left.\lim _{N \rightarrow \infty} \frac{1}{N} \ln \left|\dot{J}_{N}(q)\right|\right|_{q=e^{\frac{2 \pi i}{N}}}
$$

for the $q$-holonomic system $\left\{\dot{J}_{n}(q)\right\}$ defined by recurrence relations (28)-(29) and the initial conditions (30). That is, if we consider the polynomial $\dot{J}_{n}(q)=: \dot{Q}_{n}\left(q, q^{n}\right)$ as a function of two variables $q=e^{\frac{2 \pi i}{N}}$ and $z:=q^{n}$, then we start with the functions $\left\{\dot{Q}\left(q, q^{n}\right)\right\}_{n=1}^{3}$ taken at the points $q, q^{2}, q^{3}$, and calculating the functions $\left\{\dot{Q}\left(q, q^{n}\right)\right\}_{n=4}^{N}$ by (28)-(29) at the consecutive roots of unity $z:=q^{n}$, moving with respect to $z$ along the unit circle from $z:=q$ to $z:=q^{N}=e^{2 \pi i}$, we come to $\dot{Q}\left(q, q^{N}\right)$. To determine $\lim _{N \rightarrow \infty} \dot{Q}_{n}\left(q, q^{N}\right)$ we first find the asymptotics of the basis of solutions to the homogeneous problem (38) for $z \Subset K$ for compact sets $K$ covering $\{|z|=1\}$.

Let us note a circumstance that complicates the task. The unit circle does not lie entirely within the zone where the eigenvalues are separated: our path along $\left\{\boldsymbol{e}^{i \phi}\right\}_{\phi=0}^{2 \pi i}$ starts and ends in the zone where the eigenvalues are close to each other (the neighborhood of the point 1), and also along the path such zones appear in the neighborhoods of the points $z_{1},-1, z_{2}$, see (41) and (42). Therefore, after finding this basis in the zone of separated eigenvalues, in order to find a global (along the entire circle) asymptotic basis of solutions of the homogeneous problem, we have to find bases in four local zones close to eigenvalues (in the neighborhood of the points $1, z_{1},-1, z_{2}$ ) and the transition matrix for the basis in the zone of separated eigenvalues at the entrance and exit from local zones, where eigenvalues are close.

Finally, having at our disposal an asymptotic expansion of the general homogeneous solution along the whole unit circle, by the method of variation of constants, we find the general solution of the inhomogeneous problem (28), (29), which we match with the initial data (30). The special solution obtained in this way for the inhomogeneous problem with the help of the asymptotic basis of the homogeneous problem, we can continue from the initial zone (the neighborhood of the point $z=1$ ) along the whole circle to the finite zone which is the neighborhood of the point $z=e^{2 \pi i}$.

In the present paper, we construct the spectral curve parametrization which is needed for realisation of this plan.

\subsection{Spectral Curve Parametrization}

Theorem 2. Let $s \in \mathbb{C}$. Then for the points of the curve $\lambda(z)$, see (40), we have

$$
\left\{\begin{array}{l}
z=\frac{d+1}{d-1}, \quad d=\left(\frac{R_{3}(s)+2}{R_{3}(s)-2}\right)^{1 / 2} \\
\lambda=L(z, s)=(s+1) z^{2}-s(s+2)\left(s^{2}+s-1\right) z+s^{2}(s+2),
\end{array}\right.
$$

where

$$
R_{3}(s):=\frac{s^{3}+2 s^{2}+s-1}{s(s+1)} .
$$

Moreover, for the branches of $\lambda(z)$ we have

$$
\lambda_{j}(z)=L\left(z, s_{j}\right), \quad \text { where } \quad\left\{s_{j}\right\}_{j=0}^{2}: \quad R_{3}\left(s_{0}\right)=R_{3}\left(s_{1}\right)=R_{3}\left(s_{2}\right) .
$$

Remark. If for some $s=: s_{0}$ by (43) we have the value of the branch $\lambda_{0}=L\left(z\left(s_{0}\right), s_{0}\right)$, then to find other branches $\lambda_{1,2}$ by (45) we have to solve the cubic equation $R_{3}\left(s_{0}\right)=R_{3}(s)$ to find $s=s_{1}, s_{2}$. In practice, however, we reduce the problem to a quadratic equation as follows. In the equation in (40), we replace $z$ with its parametrization by variable $s$ (the first line in (43)). We divide the resulting expression (depending on $\lambda$ and $s$ ) by $(\lambda-L(z, s)$ ), while in the expression $L(z, s)$ (the second line in (43)) parametrization is also substituted. Symbolic calculations allow us to implement this division procedure. The result is a quadratic equation for another branches $\lambda_{1}$ and $\lambda_{2}$. 
Proof of Theorem 2. Put

$$
\mathcal{L}:=\lambda+\frac{1}{\lambda}, \quad \mathcal{Z}:=z+\frac{1}{z}
$$

If we add (40) to these equations and exclude the variables $z$ and $\lambda$, then we obtain an equation for the algebraic function $\mathcal{L}(\mathcal{Z})$ of the form

$$
\begin{gathered}
\mathcal{L}^{3}+\left(\mathcal{Z}^{5}+6 \mathcal{Z}^{2}-\mathcal{Z}^{4}-5 \mathcal{Z}^{3}-8+7 \mathcal{Z}\right) \mathcal{L}^{2} \\
+\left(16-20 \mathcal{Z}^{3}+2 \mathcal{Z}^{6}+16 \mathcal{Z}-\mathcal{Z}^{7}+3 \mathcal{Z}^{2}+8 \mathcal{Z}^{5}-9 \mathcal{Z}^{4}\right) \mathcal{L} \\
-16 \mathcal{Z}^{5}+64+34 \mathcal{Z}^{4}-48 \mathcal{Z}+2 \mathcal{Z}^{7}-4 \mathcal{Z}^{6}+45 \mathcal{Z}^{3}-88 \mathcal{Z}^{2}=0 .
\end{gathered}
$$

The algebraic curve $\mathcal{L}(\mathcal{Z})$ has genus 0 , gen $\mathcal{L}(\mathcal{Z})=0$, therefore, there is a uniformization by rational functions for it, which can be chosen as follows

$$
\left\{\begin{array}{l}
\mathcal{L}=-\frac{-1+3 s+4 s^{2}-5 s^{3}-9 s^{4}+s^{5}+4 s^{6}+s^{7}}{s^{2}} \\
\mathcal{Z}=\frac{s^{3}+2 s^{2}-1+s}{s(s+1)}
\end{array}\right.
$$

Using this uniformization and (46), we can find the parametrization of the spectral curve $\lambda(z)$. Note first the useful formula

$$
\left(\mathcal{Z}-\frac{1}{\mathcal{Z}}\right)\left(\mathcal{L}-\frac{1}{\mathcal{L}}\right)=-\frac{(s-1)(s+1)\left(s^{2}+2 s-1\right)\left(s^{3}+4 s^{2}+3 s-1\right)\left(s^{3}-s-1\right)}{s^{3}},
$$

with which we can choose the correct branch of the square root for the parametrization of coordinates of the curve using the uniformizing variable $s$ :

$$
\begin{aligned}
\lambda= & \frac{1}{2 s^{2}}\left(\sqrt{s^{3}+4 s^{2}+3 s-1}\left(1-s^{2}\right)\left(s^{2}+2 s-1\right) \sqrt{s^{3}-s-1}\right. \\
\left.+1-3 s-4 s^{2}+5 s^{3}+9 s^{4}-s^{5}-4 s^{6}-s^{7}\right) & \\
z= & \frac{\left(\sqrt{s^{3}-s-1}+\sqrt{s(s+2)^{2}-s-1}\right)^{2}}{4 s(s+1)} .
\end{aligned}
$$

Note that a different choice of branches leads to

$$
\begin{aligned}
& \frac{1}{\lambda}=\frac{-1}{2 s^{2}}\left(\sqrt{s^{3}+4 s^{2}+3 s-1}\left(1-s^{2}\right)\left(s^{2}+2 s-1\right) \sqrt{s^{3}-s-1}\right. \\
& \left.+1-3 s-4 s^{2}+5 s^{3}+9 s^{4}-s^{5}-4 s^{6}-s^{7}\right) ; \\
& \frac{1}{z}=\frac{\left(\sqrt{s^{3}-s-1}-\sqrt{s(s+2)^{2}-s-1}\right)^{2}}{4 s(s+1)} .
\end{aligned}
$$

From here, see (48), we can derive the second relation in (43):

$$
\lambda=L(z, s)=(s+1) z^{2}-s(s+2)\left(s^{2}+s-1\right) z+s^{2}(s+2),
$$

assuming that $s$ is a uniformization variable from (47).

Now we get the parametrization of the variable $z$ in the form of the first relation in (43). We know, see (46), (47), and (44), that

$$
z+\frac{1}{z}=R_{3}(s) \quad \Longleftrightarrow \quad \mathcal{Z}=R_{3}(s):=\frac{s^{3}+2 s^{2}+s-1}{s(s+1)} .
$$

The inverse Zhukovsky transform can be written as

$$
z=\frac{R_{3}}{2}+\frac{R_{3}-2}{2} \sqrt{\frac{R_{3}+2}{R_{3}-2}}, \quad \sqrt{\frac{R_{3}+2}{R_{3}-2}}=: d .
$$


Then

$$
z d-d=\frac{R_{3}}{2} d+\frac{R_{3}}{2}+1-d=\frac{R_{3}}{2}+\frac{R_{3}-2}{2} d+1=z+1
$$

that is

$$
d=\frac{z+1}{z-1} \quad \text { and } \quad z=\frac{d+1}{d-1}
$$

Note that the parametrization (43) proved in the theorem is more compact and convenient compared to (48) (in particular, to calculate $\lambda$ we use the already calculated variable $z$ ).

Finally, we comment on (45). Let's go back to (49). The rational function $R_{3}$ is of order 3 , hence

$$
\forall \mathcal{Z} \in \overline{\mathbb{C}} \quad \exists\left\{s_{k}\right\}_{k=0}^{2}: \quad \mathcal{Z}\left(s_{0}\right)=\mathcal{Z}\left(s_{1}\right)=\mathcal{Z}\left(s_{2}\right) .
$$

Theorem 2 is proved.

\section{OPEN ACCESS}

This article is licensed under a Creative Commons Attribution 4.0 International License, which permits use, sharing, adaptation, distribution and reproduction in any medium or format, as long as you give appropriate credit to the original author(s) and the source, provide a link to the Creative Commons license, and indicate if changes were made. The images or other third party material in this article are included in the article's Creative Commons license, unless indicated otherwise in a credit line to the material. If material is not included in the article's Creative Commons license and your intended use is not permitted by statutory regulation or exceeds the permitted use, you will need to obtain permission directly from the copyright holder. To view a copy of this license, visit http://creativecommons.org/licenses/by/4.0/.

\section{REFERENCES}

1. C. Adams, "Hyperbolic knots," in Handbook of Knot Theory, Ed. by W. Menasco and M. Thistlethwaite (Elsevier, Amsderdam, 2005), Chap. 1.

2. A. I. Aptekarev, "Asymptotics of orthogonal polynomials in a neighborhood of the endpoints of the interval of orthogonality," Sb. Math. 76 (1), 35-50 (1993).

3. A. I. Aptekarev and D. N. Tulyakov, "Asymptotics of Meixner polynomials and Christoffel-Darboux kernels," Trans. Mosc. Math. Soc. 73, 67-106 (2012).

4. A. I. Aptekarev and D. N. Tulyakov, "The leading term of the Plancherel-Rotach asymptotic formula for solutions of recurrence relations," Sb. Math. 205, 1696-1719 (2014).

5. A. I. Aptekarev and D. N. Tulyakov, "Asymptotical basis of solutions of $q$-recurrence relations outside of the zone of clustering eigenvalues," KIAM Preprint No. 159 (Keldysh Inst. Appl. Math., Moscow, 2018). https://doi.org/10.20948/prepr-2018-159

6. A. I. Aptekarev, V. Kalyagin, G. Lopez Lagomasino, and I. A. Rocha, "On the limit behavior of recurrence coefficients for multiple orthogonal polynomials," J. Approx. Theory 139, 346-370 (2006).

7. I. N. Bernstein, "Modules over a ring of differential operators, study of the fundamental solutions of equations with constant coefficients," Function. Anal. Appl. 5, 89-101 (1971).

8. I. N. Bernstein, "The analytic continuation of generalized functions with respect to a parameter," Function. Anal. Appl. 6, 273-285 (1972).

9. G. Burde and H. Zieschang, Knots, Vol. 5 of de Gruyter Studies in Mathematics (Gruyter, Berlin, 1985).

10. O. Costin and R. Costin, "Rigorous WKB for finite-order linear recurrence relations with smooth coefficients," SIAM J. Math. Anal. 27, 110-134 (1996).

11. D. Cooper, D. M. Culler, H. Gillet, D. Long, and P. Shalen, "Plane curves associated to character varieties of 3-manifolds," Invent. Math. 118, 47-84 (1994).

12. S. Garoufalidis and T. T. Q. Lê, "The colored Jones function is $q$-holonomic," Geom. Topol. 9, 1253-1293 (2005).

13. S. Garoufalidis, "On the characteristic and deformation varieties of a knot," in Proceedings of the CassonFest, Vol. 7 of Geometry and Topology Monographs (Math. Sci. Publ., 2004), pp. 291-309.

14. S. Garoufalidis and J. S. Geronimo, "Asymptotics of $q$-difference equations," in Primes and Knots, Ed. by T. Kohno and M. Morishita, Vol. 416 of Contemporary Mathematics (Am. Math. Soc., 2006), pp. 83-114.

15. S. Garoufalidis and T. T. Q. Lê, "On the volume conjecture for small angles," arXiv: math.GT/0502163 (2005). 
16. S. Garoufalidis and T. Lê, "Asymptotics of the colored Jones function of a knot," Geom. Topol. 15, 2135-2180 (2011).

17. S. Garoufalidis and C. Koutschan, "Irreducibility of $q$-difference operators and the knot $7_{4}$," Algebr. Geom. Topol. 13, 3261-3286 (2013).

18. S. Garoufalidis and X. Sun, "The non-commutative $A$-polynomial of twist knots," J. Knot Theory Ramif. 19, 1571-1595 (2010).

19. H. Gieseking, "Analytische Untersuchungen ueber topologische Gruppen," Thesis (privately printed, Muenster, 1912); W. Magnus, Noneuclidean Tesselations and Their Groups (Academic, New York, 1974), p. 153.

20. M. Gromov, "Volume and bounded cohomology," Publ. Math., Inst. Hautes Etud. Sci. 56, 5-100 (1982).

21. S. Gukov, "Three-dimensional quantum gravity, Chern-Simons theory, and the $A$-polynomial," Commun. Math. Phys. 255, 577-627 (2005).

22. K. Habiro, "On the colored Jones polynomials of some simple links," in Recent Progress towards the Volume Conjecture (Kyoto, 2000), Surikaisekikenkyusho Kokyuroku 1172, 34-43 (2000).

23. K. Habiro, "On the quantum $\mathfrak{s l}_{2}$ invariants of knots and integral homology spheres," in Invariants of Knots and 3-manifolds, Kyoto, 2001, Vol. 4 of Geometry and Topology Monographs (Geom. Topol. Publ., 2004), pp. 55-68.

24. K. Hikami, "Volume conjecture and asymptotic expansion of $q$-series," Exp. Math. 12, 319-337 (2003).

25. K. Hikami, "Difference equation of the colored Jones polynomial for torus knot," Int. J. Math. 15, 959-965 (2004).

26. V. F. R. Jones, "Polynomial invariants of knots via von Neumann algebras," Bull. Am. Math. Soc. 12, 103$111(1985)$.

27. V. Jones, "Hecke algebra representation of braid groups and link polynomials," Ann. Math. 126, 335-388 (1987).

28. R. M. Kashaev, "A link invariant from quantum dilogarithm,” Mod. Phys. Lett. A 10, 1409-1418 (1995).

29. R. M. Kashaev, "The hyperbolic volume of knots from the quantum dilogarithm," Lett. Math. Phys. 39, 269275 (1997).

30. R. M. Kashaev and O. Tirkkonen, "A proof of the volume conjecture on torus knots," J. Math. Sci. (N.Y.) 115, 2033-2036 (2003).

31. L. Kauffman, "States models and the Jones polynomial," Topology 26, 395-407 (1987).

32. T. T. Q. Le, "Quantum invariants of 3-manifolds: Integrality, splitting, and perturbative expansion," Topol. Appl. 127, 125-152 (2003).

33. T. Le, "The colored Jones polynomial and the A-polynomial of knots," Adv. Math. 207, 782-804 (2006).

34. T. Le and A. Tran, "On the AJ conjecture for knots," Indiana Univ. Math. J. 64, 1103-1151 (2015).

35. T. Le and X. Zhang, "Character varieties, $A$-polynomials, and the $A J$ conjecture," Algebr. Geom. Topol. 17, $157-188(2017)$.

36. C. Livingston and A. H. Moore, KnotInfo: Table of Knot Invariants. http://knotinfo.math.indiana.edu. Accessed April 22, 2021.

37. G. Masbaum, "Skein-theoretical derivation of some formulas of Habiro," Algebr. Geom. Topol. 3, 537-556 (2003).

38. J. W. Milnor, “Hyperbolic geometry: The first 150 year,” Bull. Am. Math. Soc. 6, 232-248 (1982).

39. H. Murakami and J. Murakami, "The colored Jones polynomials and the simplicial volume of a knot," Acta Math. 186, 85-104 (2001).

40. H. Murakami and Y. Yokota, Volume Conjecture for Knots, Vol. 30 of Springer Briefs in Mathematical Physics (Springer, Berlin, 2018).

41. T. Ohtsuki, "On the asymptotic expansion of the Kashaev invariant of the $5_{2}$ knot," Quantum Topol. 7, 669735 (2016).

42. T. Ohtsuki and Y. Yokota, "On the asymptotic expansions of the Kashaev invariant of the knots with 6 crossings," Math. Proc. Cambridge Philos. Soc. 165, 287-339 (2018).

43. T. Ohtsuki, "On the asymptotic expansions of the Kashaev invariant of hyperbolic knots with seven crossings," Int. J. Math. 28, 1750096 (2017).

44. T. Ohtsuki and T. Takata, "On the Kashaev invariant and the twisted Reidemeister torsion of two-bridge knots," Geom. Topol. 19, 853-952 (2015).

45. P. Paule and A. Riese, "A mathematica $q$-analogue of Zeilberger's algorithm based on an algebraically motivated approach to $q$-hypergeometric telescoping," in Special Functions, $q$-Series and Related Topics, Fields Inst. Commun. 14, 179-210 (1997).

46. M. Petkovšek, H. S. Wilf and D. Zeilberger, $A=B$ (AK Peters, Wellesley, 1996).

47. N. Yu. Reshetikhin and V. G. Turaev, "Invariants of 3-manifolds via link polynomials and quantum groups," Invent. Math. 103, 547-597 (1991).

48. R. Riley, "A quadratic parabolic group,” Math. Proc. Cambridge Philos. Soc. 77, 281-288 (1975). 
49. D. Rolfsen, Knots and Links, Vol. 7 of Mathematics Lecture Series (Publish or Perish, Houston, 1990).

50. T. Takata, "The colored Jones polynomial and the $A$-polynomial for twist knots," arXiv: mathGT/0401068 (2004).

51. W. P. Thurston, The Geometry and Topology of Three-Manifolds, Electronic Version 1.1. http://www.msri.org/publications/books/gt3m/. Accessed March 2002.

52. W. P. Thurston, "Three-dimensional manifolds, Kleinian groups and hyperbolic geometry," Bull. Am. Math. Soc. (N.S.) 6, 357-381 (1982).

53. A. T. Tran, "Some conjectures about the colored Jones polynomial," in Intelligence of Low-dimensional Topology, Vol. 2052 of Kyoto University Research Information Repository (2017), pp. 74-81. repository.kulib.kyoto-u.ac.jp.

54. A. Tran, "Proof of a stronger version of the AJ conjecture for torus knots," Algebr. Geom. Topol. 13, 609-624 (2013).

55. D. N. Tulyakov, "Local asymptotics of the ratio of orthogonal polynomials in the neighbourhood of an endpoint of the support of the orthogonality measure," Sb. Math. 192, 299-321 (2001).

56. D. N. Tulyakov, "Difference equations having bases with powerlike growth which are perturbed by a spectral parameter," Sb. Math. 200, 753-781 (2009).

57. D. N. Tulyakov, "Plancherel-Rotach type asymptotics for solutions of linear recurrence relations with rational coefficients," Sb. Math. 201, 1355-1402 (2010).

58. V. G. Turaev, "The Yang-Baxter equation and invariants of links," Invent. Math. 92, 527-553 (1988).

59. V. G. Turaev, Quantum Invariants of Knots and 3-Manifolds, Vol. 18 of de Gruyter Studies in Mathematics (Walter de Gruyter, Berlin, 1994).

60. R. van der Veen, "Proof of the volume conjecture for Whitehead chains," Acta Math. Vietnam 33, 421-431 (2008).

61. H. S. Wilf and D. Zeilberger, "An algorithmic proof theory for hypergeometric (ordinary and " $q$ ") multisum/integral identities," Invent. Math. 108, 575-633 (1992).

62. D. Zeilberger, "A holonomic systems approach to special functions identities," J. Comput. Appl. Math. 32, 321-368 (1990).

63. H. Zheng, "Proof of the volume conjecture for Whitehead doubles of a family of torus knots," Chin. Ann. Math., Ser. B 28, 375-388 (2007). 